\title{
Modifications of Kukasiewicz's intuitionistic fuzzy implication
}

\author{
Alžbeta Michalíková ${ }^{1,2}$, Eulalia Szmidt ${ }^{3,4}$ and Peter Vassilev ${ }^{5}$ \\ ${ }^{1}$ Faculty of Natural Sciences, Matej Bel University \\ Tajovského 40, Banská Bystrica, Slovakia \\ e-mail: alzbeta.michalikova@umb.sk \\ ${ }^{2}$ Mathematical Institute, Slovak Academy of Sciences \\ Ďumbierska 1, Banská Bystrica, Slovakia \\ ${ }^{3}$ Systems Research Institute, Polish Academy of Sciences \\ Newelska 6, 01 - 447 Warsaw, Poland \\ e-mail:s zmidt@ibspan.waw.pl \\ ${ }^{4}$ Warsaw School of Information Technology \\ ul. Newelska 6, 01 - 447 Warsaw, Poland \\ ${ }^{5}$ Department of Bioinformatics and Mathematical Modelling, \\ Institute of Biophysics and Biomedical Engineering, Bulgarian Academy of Sciences \\ 105 Acad. Georgi Bonchev Str., 1113 Sofia, Bulgaria \\ e-mail: peter.vassilev@gmail.com
}

Received: 7 May 2021

Accepted: 27 August 2021

\begin{abstract}
In [6], G. Klir and B. Yuan named after J. Łukasiewicz the implication $p \rightarrow q=$ $\min (1, p+q)$. In a series of papers, 198 different intuitionistic fuzzy implications have been introduced, and their basic properties have been studied. Here we introduce six new implications which are modifications of Łukasiewicz's intuitionistic fuzzy implication, and we describe and prove some of their properties.
\end{abstract}

Keywords: Intuitionistic fuzzy implication, Intuitionistic fuzzy set, Łukasiewicz's fuzzy implication.

2020 Mathematics Subject Classification: $03 E 72$. 


\section{Introduction}

In [1,2], Łukasiewicz's intuitionistic fuzzy implication was introduced and some of its basic properties were studied. It is based on Łukasiewicz's fuzzy implication that has the following form (see, e.g., [6]):

$$
p \rightarrow q=\min (1, p+q) .
$$

Here, following the scheme from [2,4,5], we will construct eight new implications, related to the Eukasiewicz's intuitionistic fuzzy implication but we will observe that two of them coincide and one is trivial as it permanently yields in result a constant. Therefore, of these eight six are the new implications which will present and study in details.

\section{Preliminaries}

In [3], K. Atanassov, E. Szmidt and J. Kacprzyk defined the object $\langle a, b\rangle$, with $a, b, a+b \in[0,1]$, as Intuitionistic Fuzzy Pair (IFP).

Let everywhere below for IFPs $x$ and $y$ :

$$
\begin{aligned}
& x=\langle a, b\rangle, \\
& y=\langle c, d\rangle,
\end{aligned}
$$

where $a, b, c, d \in[0,1], a+b \leq 1, c+d \leq 1$. For the IFPs, in [3], different operations and relations have been defined. For our aims, we will remind the definitions of only three of these relations and one operation:

$$
\begin{aligned}
x & \leq y \text { if and only if } a \leq c \text { and } b \geq d, \\
x & \geq y \text { if and only if } y \leq x, \\
x & =y \text { if and only if } x \leq y \text { and } y \leq x, \\
\neg x & =\langle b, a\rangle .
\end{aligned}
$$

\section{Main results}

Łukasiewicz's intuitionistic fuzzy implication (see $[1,2]$ ) is defined by

$$
x \rightarrow_{L} y=\langle\min (1, b+c), \max (0, a+d-1)\rangle .
$$

The (standard) intuitionistic fuzzy modal operators over IFPs (see, e.g., [2]) are:

$$
\begin{aligned}
& \square x=\langle a, 1-a\rangle, \\
& \diamond x=\langle 1-b, b\rangle .
\end{aligned}
$$

The intuitionistic fuzzy modal operators of second type over IFPs (see [2]) are:

$$
\begin{aligned}
& \boxplus x=\left\langle\frac{a}{2}, \frac{b+1}{2}\right\rangle, \\
& \boxplus x=\left\langle\frac{a+1}{2}, \frac{b}{2}\right\rangle .
\end{aligned}
$$


Now, using the idea from [4] and the logical scheme (see, e.g. $[2,5]$ )

$$
x \rightarrow_{*} y=M_{1} x \rightarrow_{L} M_{2} y,
$$

where $M_{1}, M_{2} \in\{\square, \diamond\}$, we define the following new implications:

$$
\begin{aligned}
x \rightarrow_{1} y & =\square x \rightarrow_{L} \diamond y=\langle a, 1-a\rangle \rightarrow\langle 1-d, d\rangle \\
& =\langle\min (1,2-a-d), \max (0, a+d-1)\rangle ; \\
x \rightarrow_{2} y & =\square x \rightarrow_{L} \square y=\langle a, 1-a\rangle \rightarrow\langle c, 1-c\rangle \\
& =\langle\min (1,1-a+c), \max (0, a-c)\rangle ; \\
x \rightarrow_{3} y & =\diamond x \rightarrow_{L} \diamond y=\langle 1-b, b\rangle \rightarrow\langle 1-d, d\rangle \\
& =\langle\min (1,1+b-d), \max (0, d-b)\rangle ; \\
x \rightarrow_{4} y & =\diamond x \rightarrow_{L} \square y=\langle 1-b, b\rangle \rightarrow\langle c, 1-c\rangle \\
& =\langle\min (1, b+c), \max (0,1-b-c)\rangle .
\end{aligned}
$$

For these four new implications we can prove the following assertions.

Theorem 1. For every two IFPs $x$ and $y$

$$
x \rightarrow_{1} y \geq\left\{\begin{array}{l}
x \rightarrow_{2} y \\
x \rightarrow_{3} y
\end{array}\right\} \geq x \rightarrow_{4} y .
$$

Proof. Let the two IFPs $x$ and $y$ be given. Then from the inequalities

$$
\begin{aligned}
\min (1,2-a-d) & \geq \min (1,1-a+c), \\
\min (1,2-a-d) & \geq \min (1,1+b-d), \\
\min (1,1-a+c) & \geq \min (1, b+c), \\
\min (1,1+b-d) & \geq \min (1, b+c), \\
\max (0, a+d-1) & \leq \max (0, a-c), \\
\max (0, a+d-1) & \leq \max (0, d-b), \\
\max (0, a-c) & \leq \max (0,1-b-c), \\
\max (0, d-b) & \leq \max (0,1-b-c) .
\end{aligned}
$$

the validity of Theorem 1 follows.

In contrast to [4], in the general case, the relations in the form

$$
\neg x \rightarrow_{i} \neg y=\neg\left(x \rightarrow_{j} y\right)
$$

are not valid for $1 \leq i, j \leq 4$ 
Indeed, for $i=1$ we obtain:

$$
\begin{aligned}
\neg x \rightarrow_{1} \neg y & =\neg\langle a, b\rangle \rightarrow_{1} \neg\langle c, d\rangle=\langle b, a\rangle \rightarrow_{1}\langle d, c\rangle \\
& =\langle\min (1,2-b-c), \max (0, b+c-1)\rangle \\
& =\neg\langle\max (0, b+c-1), \min (1,2-b-c)\rangle,
\end{aligned}
$$

which is not equal to any $\neg\left(x \rightarrow_{j} y\right)$ with $1 \leq j \leq 4$.

Similarly to [4], if for the scheme $(*)$ we use $M_{1}, M_{2} \in\{\boxplus, \bigotimes\}$, we will receive the following four new intuitionistic fuzzy implications:

$$
\begin{aligned}
& x \rightarrow_{5} y=\boxplus x \rightarrow_{L} \boxplus y=\left\langle\frac{a}{2}, \frac{b+1}{2}\right\rangle \rightarrow_{L}\left\langle\frac{c}{2}, \frac{d+1}{2}\right\rangle \\
& =\left\langle\min \left(1, \frac{b+1}{2}+\frac{c}{2}\right), \max \left(0, \frac{a}{2}+\frac{d+1}{2}-1\right)\right\rangle \\
& =\left\langle\min \left(1, \frac{b+c+1}{2}\right), \max \left(0, \frac{a+d-1}{2}\right)\right\rangle \text {; } \\
& x \rightarrow_{6} y=\bigotimes x \rightarrow_{L} \boxplus y=\left\langle\frac{a+1}{2}, \frac{b}{2}\right\rangle \rightarrow_{L}\left\langle\frac{c}{2}, \frac{d+1}{2}\right\rangle \\
& =\left\langle\min \left(1, \frac{b}{2}+\frac{c}{2}\right), \max \left(0, \frac{a+1}{2}+\frac{d+1}{2}-1\right)\right\rangle \\
& =\left\langle\min \left(1, \frac{b+c}{2}\right), \max \left(0, \frac{a+d}{2}\right)\right\rangle \\
& =\left\langle\frac{b+c}{2}, \frac{a+d}{2}\right\rangle \text {; } \\
& x \rightarrow_{7} y=\boxplus x \rightarrow_{L} \bigotimes y=\left\langle\frac{a}{2}, \frac{b+1}{2}\right\rangle \rightarrow_{L}\left\langle\frac{c+1}{2}, \frac{d}{2}\right\rangle \\
& =\left\langle\min \left(1, \frac{b+1}{2}+\frac{c+1}{2}\right), \max \left(0, \frac{a}{2}+\frac{d}{2}-1\right)\right\rangle \\
& =\left\langle\min \left(1, \frac{b+c+2}{2}\right), \max \left(0, \frac{a+d-2}{2}\right)\right\rangle \\
& =\langle 1,0\rangle \text {; } \\
& x \rightarrow_{8} y=\bigotimes x \rightarrow_{L} \bigotimes y=\left\langle\frac{a+1}{2}, \frac{b}{2}\right\rangle \rightarrow_{L}\left\langle\frac{c+1}{2}, \frac{d}{2}\right\rangle \\
& =\left\langle\min \left(1, \frac{b}{2}+\frac{c+1}{2}\right), \max \left(0, \frac{a+1}{2}+\frac{d}{2}-1\right)\right\rangle \\
& =\left\langle\min \left(1, \frac{b+c+1}{2}\right), \max \left(0, \frac{a+d-1}{2}\right)\right\rangle \text {. }
\end{aligned}
$$

Obviously, implication $\rightarrow_{7}$ is trivial, because its result is always a constant, while implications $\rightarrow_{5}$ and $\rightarrow_{8}$ coincide and hence, we can only work with the first of them, $\rightarrow_{5}$.

Now, we formulate Theorem 2, which can be proved in the same manner as Theorem 1, hence the proof is skipped. 
Theorem 2. For every two IFPs $x$ and $y$

$$
x \rightarrow_{5} y \geq x \rightarrow_{6} y .
$$

We directly check the validity of the following equalities.

$$
\begin{aligned}
& \langle 0,1\rangle \rightarrow_{i}\langle 0,1\rangle=\left\{\begin{array}{ll}
\langle 1,0\rangle, & \text { for } i=1,2,3,4,5 \\
\left\langle\frac{1}{2}, \frac{1}{2}\right\rangle, & \text { for } i=6
\end{array} ;\right. \\
& \langle 0,1\rangle \rightarrow_{i}\langle 0,0\rangle=\left\{\begin{array}{ll}
\langle 1,0\rangle, & \text { for } i=1,2,3,4,5 \\
\left\langle\frac{1}{2}, 0\right\rangle, & \text { for } i=6
\end{array} ;\right. \\
& \langle 0,1\rangle \rightarrow_{i}\langle 1,0\rangle=\langle 1,0\rangle, \text { for each } i=1, \ldots, 6 \text {; } \\
& \langle 0,0\rangle \rightarrow_{i}\langle 0,1\rangle=\left\{\begin{array}{ll}
\langle 1,0\rangle, & \text { for } i=1,2 \\
\langle 0,1\rangle, & \text { for } i=3,4 \\
\left\langle\frac{1}{2}, 0\right\rangle, & \text { for } i=5 \\
\langle 0,0\rangle, & \text { for } i=6
\end{array} ;\right. \\
& \langle 0,0\rangle \rightarrow_{i}\langle 0,0\rangle=\left\{\begin{array}{ll}
\langle 1,0\rangle, & \text { for } i=1,2,3 \\
\langle 0,1\rangle, & \text { for } i=4 \\
\left\langle\frac{1}{2}, 0\right\rangle, & \text { for } i=5 \\
\langle 0,0\rangle, & \text { for } i=6
\end{array} ;\right. \\
& \langle 0,0\rangle \rightarrow_{i}\langle 1,0\rangle=\left\{\begin{array}{ll}
\langle 1,0\rangle, & \text { for } i=1,2,3,4,5 \\
\left\langle\frac{1}{2}, 0\right\rangle, & \text { for } i=6
\end{array} ;\right. \\
& \langle 1,0\rangle \rightarrow_{i}\langle 0,1\rangle=\left\{\begin{array}{ll}
\langle 0,1\rangle, & \text { for } i=1,2,3,4,6 \\
\left\langle\frac{1}{2}, \frac{1}{2}\right\rangle, & \text { for } i=5
\end{array} ;\right. \\
& \langle 1,0\rangle \rightarrow_{i}\langle 0,0\rangle=\left\{\begin{array}{ll}
\langle 1,0\rangle, & \text { for } i=1,3 \\
\langle 0,1\rangle, & \text { for } i=2,4 \\
\left\langle\frac{1}{2}, 0\right\rangle, & \text { for } i=5 \\
\left\langle 0, \frac{1}{2}\right\rangle, & \text { for } i=6
\end{array} ;\right. \\
& \langle 1,0\rangle \rightarrow_{i}\langle 1,0\rangle=\left\{\begin{array}{ll}
\langle 1,0\rangle, & \text { for } i=1,2,3,4,5 \\
\left\langle\frac{1}{2}, \frac{1}{2}\right\rangle, & \text { for } i=6
\end{array} .\right.
\end{aligned}
$$

Following [2] and using, e.g. [7], we mention that

$$
\neg x=x \rightarrow\langle 0,1\rangle .
$$


Therefore, the new implications generate the following negations:

$$
\begin{aligned}
& \neg_{1} x=x \rightarrow_{1}\langle 0,1\rangle=\langle 1-a, a\rangle ; \\
& \neg_{2} x=x \rightarrow_{2}\langle 0,1\rangle=\langle 1-a, a\rangle ; \\
& \neg_{3} x=x \rightarrow_{3}\langle 0,1\rangle=\langle b, 1-b\rangle ; \\
& \neg_{4} x=x \rightarrow_{4}\langle 0,1\rangle=\langle b, 1-b\rangle ; \\
& \neg_{5} x=x \rightarrow_{5}\langle 0,1\rangle=\left\langle\frac{b+1}{2}, \frac{a}{2}\right\rangle ; \\
& \neg_{6} x=x \rightarrow_{6}\langle 0,1\rangle=\left\langle\frac{b}{2}, \frac{a+1}{2}\right\rangle .
\end{aligned}
$$

We see immediately that for each IFP $x$

$$
\neg_{1} x=\neg_{2} x \geq \neg_{3} x=\neg_{4} x
$$

and

$$
\neg_{5} x>\neg_{6} x .
$$

It is important to mention that the constructed here implications are new ones, while negations $\neg_{1}$ and $\neg_{2}$ coincide with negation $\neg_{8}$ from [2], negations $\neg_{3}$ and $\neg_{4}-$ with negation $\neg_{4}$ from [2], negations $\neg_{6}-$ with negation $\neg_{35}$ from [2] and only negation $\neg_{5}$ is a new one.

In [6], G. Klir and B. Yuan give nine axioms for fuzzy implication. In [2], K. Atanassov pre-formulated them for the case of intuitionistic fuzziness.

Let

$$
\begin{aligned}
& O^{*}=\langle 0,1\rangle, \\
& E^{*}=\langle 1,0\rangle .
\end{aligned}
$$

The IFP is a tautology iff $a=1$ and $b=0$, while it is an Intuitionistic Fuzzy Tautology (IFT) iff $a \geq b$.

Klir and Yuan's axioms are:

$$
\begin{aligned}
& \text { Axiom } \boldsymbol{A 1}(\forall x, y)(\text { if } x \leq y \text {, then }(\forall z)(x \rightarrow z \geq y \rightarrow z))), \\
& \text { Axiom } \boldsymbol{A 2}(\forall x, y)(\text { if } x \leq y \text {, then }(\forall z)(z \rightarrow x \leq z \rightarrow y))), \\
& \text { Axiom } \boldsymbol{A 3}(\forall y)\left(O^{*} \rightarrow y=E^{*}\right), \\
& \text { Axiom } \boldsymbol{A 4}(\forall y)\left(E^{*} \rightarrow y=y\right), \\
& \text { Axiom } \boldsymbol{A 5}(\forall x)\left(x \rightarrow x=E^{*}\right), \\
& \text { Axiom } \boldsymbol{A 6}(\forall x, y, z)(x \rightarrow(y \rightarrow z)=y \rightarrow(x \rightarrow z)), \\
& \text { Axiom } \boldsymbol{A} 7(\forall x, y)\left(x \rightarrow y=E^{*} \text { iff } x \leq y\right),
\end{aligned}
$$


Axiom $\boldsymbol{A 8}(\forall x, y)(x \rightarrow y=\neg y \rightarrow \neg x)$

Axiom $A 9 I$ is a continuous function.

In [2], having in mind the specific forms of the intuitionistic fuzzy implications, Atanassov modified five of these axioms, as follows.

Axiom $\boldsymbol{A 3}^{*}(\forall y)\left(O^{*} \rightarrow y\right.$ is an IFT),

Axiom $\boldsymbol{A 4}^{*}(\forall y)\left(E^{*} \rightarrow y \leq y\right)$,

Axiom $\boldsymbol{A 5}^{*}(\forall x)(x \rightarrow x$ is an IFT),

Axiom $\boldsymbol{A 7}^{*}(\forall x, y)\left(\right.$ if $x \leq y$, then $\left.x \rightarrow y=E^{*}\right)$,

Axiom $\boldsymbol{A 8}^{*}(\forall x, y)(x \rightarrow y=\neg(\neg(\neg y \rightarrow \neg x)))$.

The proofs of the following theorems are similar to that of Theorem 1 .

Theorem 3. For every three IFPs $x, y$ and $z$

(a) implications $\rightarrow_{1}$ and $\rightarrow_{3}$ satisfy Axioms $A 1, A 2, A 3, A 3^{*}, A 5, A 5^{*}, A 6, A 7^{*}$;

(b) implication $\rightarrow_{2}$ satisfies Axioms $A 1, A 2, A 3, A 3^{*}, A 4^{*}, A 5, A 5^{*}, A 6, A 7^{*}, A 8, A 8^{*}$;

(c) implication $\rightarrow_{4}$ satisfies Axioms $A 1, A 2, A 3, A 3^{*}, A 4^{*}, A 6$;

(d) implication $\rightarrow_{5}$ satisfies Axioms $A 1, A 2, A 3, A 3^{*}$;

(e) implication $\rightarrow_{6}$ satisfies only Axiom $A 4^{*}$

as tautologies.

Theorem 4. For every three IFPs $x, y$ and $z$

(a) implications $\rightarrow_{1}$ and $\rightarrow_{3}$ satisfy Axioms $A 1, A 2, A 3, A 3^{*}, A 5, A 5^{*}, A 6, A 7^{*}$;

(b) implication $\rightarrow_{2}$ satisfies Axioms $A 1, A 2, A 3, A 3^{*}, A 4^{*}, A 5, A 5^{*}, A 6, A 7^{*}, A 8, A 8^{*}$;

(c) implication $\rightarrow_{4}$ satisfies Axioms $A 1, A 2, A 3, A 3^{*}, A 4^{*}, A 6$;

(d) implication $\rightarrow_{5}$ satisfies Axioms $A 1, A 2, A 3, A 3^{*}, A 5^{*}, A 7^{*}$;

(e) implication $\rightarrow_{6}$ satisfies Axioms $A 1, A 2, A 3^{*}, A 4^{*}, A 5^{*}$

as IFTs.

\section{Conclusion}

In the present paper, six new intuitionistic fuzzy implications and the negations generated by them are introduced and some of their properties are studied. In a next research, we will check which axioms of the Kolmogorov's, Łukasiewicz and Tarski's axioms for implications and which intuitionistic logic axioms are valid for the new implications. 


\section{Acknowledgements}

This first and the third authors are grateful for the support provided under the joint research project of the Bulgarian Academy of Sciences and the Slovak Academy of Sciences, entitled "Generation and Applications of Probabilistic and Intuitionistic Fuzzy Models of Uncertainty".

This second and the third authors are grateful grateful for the support provided under the joint research project of the Bulgarian Academy of Sciences and the Polish Academy of Sciences, entitled "Intuitionistic Fuzzy Sets - Theory and Applications in Medicine, Economy and other areas".

\section{References}

[1] Atanassov, K. (2006). On some intuitionistic fuzzy implications. Comptes Rendus de l'Academie bulgare des Sciences, 59(1), 19-24.

[2] Atanassov, K. (2017). Intuitionistic Fuzzy Logics, Springer, Cham, 2017.

[3] Atanassov, K., Szmidt, E., \& Kacprzyk, J. (2013). On intuitionistic fuzzy pairs. Notes on Intuitionistic Fuzzy Sets, 19(3), 1-13.

[4] Dworniczak, P., Atanassova, L., \& Angelova, N. Modal type of weak intuitionistic fuzzy implications generated by the operation $\triangle$. Cybernetics and Information Technologies (submitted)

[5] Feys, R. (1965). Modal Logics. Gauthier-Villars, Paris.

[6] Klir, G., \& Yuan, B. (1995). Fuzzy Sets and Fuzzy Logic. Prentice Hall, New Jersey.

[7] Mendelson, E. (1964). Introduction to Mathematical Logic, Princeton, NJ: D. Van Nostrand; Fourt Ed. 2001. 